Katharina Hörmann", Elisabeth Koblmiller ${ }^{* *}$ \& Lukas Tröscher ${ }^{* *}$

\title{
(De-)Konstruktion des klassischen Familienbildes im politisch bildenden GW-Unterricht
}

\author{
*katharina.hoermann@gmx.net, Unterrichtspraktikantin am BG/BRG Zwettl \\ ** e.koblmiller@gmail.com, Studentin am Institut für Geographie und Regionalforschung, Universität Wien \\ *** lukas.troescher@gmx.at, Student am Institut für Geographie und Regionalforschung, Universität Wien
}

eingereicht am 07.02.2016; akzeptiert am 15.12.2016

\begin{abstract}
Familien stellen eine interessante Analysequelle für den GW-Unterricht dar, weil anhand unterschiedlicher Familienformen demographische Sachverhalte realitätsnah veranschaulicht werden können, zudem werden sie wesentlich von soziokulturellen und ökonomischen Faktoren geprägt. In der von uns konzipierten und eingehend erläuterten Unterrichtssequenz soll vermittelt werden, dass tradierte Normvorstellungen des österreichischen Familienbilds kritisch hinterfragt werden müssen, da durch die Pluralisierung der Lebensstile heutzutage eine große Vielfalt an entsprechenden Konstellationen besteht.
\end{abstract}

Keywords: Demographie, Dekonstruktion, Familie, Familienbilder, Konstruktivismus

\section{The (de-)construction of the traditional image of the family in geography and economics}

In this article, a lesson sequence shall be presented which deals with the (de-)construction of traditional family representations in geography and economics. In course of the two lessons, the students shall not only critically reflect on traditional representations of the family consisting of mother, father and their biological children. More importantly, they should also increase their awareness towards the existence of alternative, non-traditional family configurations.

Keywords: demography, deconstruction, family, family configurations, constructivism

\section{$1 \quad$ Einleitung}

Das Familienbild in Österreich ist einem starken Wandel ausgesetzt. Ist in der zweiten Hälfte des 20. Jahrhunderts noch das heterosexuelle Ehepaar mit Kind die gesellschaftliche Norm, existieren gegenwärtig vielfältige Möglichkeiten der Familiengestaltung, was auf soziokulturelle, ökonomische und politische Veränderungen zurückzuführen ist. Trotzdem wird von kirchlichen Institutionen, von verschiedenen politischen Parteien und in der Werbung meist oben genanntes, traditionelles und im kollektiven Gedächtnis häufig noch immer idealisiertes Familienbild präsentiert und als soziale Norm suggeriert.

Es liegt in unserem Interesse als GW-Lehrende derartige Suggestionen zu relativieren und im Zuge des Unterrichts den tatsächlichen Status Quo von Familien in Österreich zu zeigen. Zudem soll die Vielfalt an Formen der Familienkonstellationen verdeutlicht werden. Einerseits erachten wir das als wichtig, weil die Institution Familie entsprechend dem RaumMensch-Paradigma des GW-Unterrichts von gesellschaftlichen, ökonomischen und politischen Prozessen mitgestaltet wird und daher eine thematisch relevante wie interessante Analysequelle darstellt. Andererseits sollen die Lernenden zur Erkenntnis gelangen, dass familiäre Fragestellungen ihr Leben wesentlich beeinflussen und diese ihre Zukunft als wesentliche Elemente des täglichen Daseins mitgestalten werden.

Inspiriert von einem kritisch-emanzipatorischen Vermittlungsinteresse (vgl. Vielhaber 1999) haben wir daher ein Unterrichtsbeispiel erstellt, welches tradierte Familienbilder analysiert bzw. dekonstruiert und darstellt, auf welch vielfältige Weise die Institution Familie heutzutage realisiert werden kann. Diese Unterrichtssequenz durften wir im Zuge des fachdidaktischen Praktikums in GW in einer ersten Klasse (9. Schulstufe) einer Handelsakademie (im Weiteren: HAK) unterrichten. Die entsprechenden fachwissenschaftlichen und fachdidaktischen Überlegungen, die 
Ergebnisse einer konkreten Umsetzung des Beispiels sowie unser Resümee finden sich auf den folgenden Seiten.

\section{Theoretische Grundlagen}

\subsection{Der Terminus Familie im fachwissenschaftli- chen Kontext}

Denken Sie an den Begriff Familie! Welches Bild haben Sie dabei vor Augen?

Wenn man den Begriff Familie verwendet, bezieht man sich oft auf das „konventionelle“ Kernfamilienmodell, welches aus einem verheirateten, heterosexuellem Paar mit seinen leiblichen Kindern besteht. Auch ein Experiment in einer 9. Schulstufe in Wien, bei dem 25 Schülerinnen und Schüler eine Familie aufzeichnen sollten, zeigte, dass bei vielen das konventionelle Kernfamilienmodell stark im kognitiven Bewusstsein verankert ist, während andere Familienkonstellationen weniger präsent sind. Dies kann vermutlich darauf zurückgeführt werden, dass die Schülerinnen und Schüler zumeist auch in einer Familie aufwachsen, die dem klassischen Familienbild entspricht. Andere Familienkonstellationen hingegen stehen in einem starken Kontrast zu den erlebten Familiensituationen und sind daher in der Wahrnehmung der Schülerinnen und Schüler seltener vorhanden.

Familie ist ein selbstverständlicher Begriff, da jede und jeder seine persönlichen Erfahrungen und Assoziationen mit dem Begriff in Verbindung setzt. Wenn man laut Lüscher (o.J. zitiert in Beham 1989, 9) von Familie spricht, passiert dies meist „vor dem Hintergrund persönlicher Erfahrungen, die ihrerseits von gesellschaftlichen Auffassungen und deren historischen Hintergründen abhängen“ (ebd.). Im Zuge der Sozialisierung bekommt man eine Vorstellung, wie Familie funktioniert oder auszusehen hat. Diese Vorstellungen variieren in den verschiedenen Kulturkreisen und so unterscheiden sich auch die Ansichten, welche Familienkonzepte als „legitim“ oder „normal“ angesehen werden (vgl. ÖIF 1999).

Die Ansichten können aber auch innerhalb der Kulturkreise bzw. im zeitlichen Verlauf stark variieren. 1973 definierte Feichtinger den Begriff Familie folgendermaßen: „Eine Familie im Sinne der Familienstatistik ist zunächst die in einem Haushalt zusammenlebende Personengruppe, wobei ein direktes Abstammungsverhältnis der zugehörigen Personen gegeben sein muß" (Feichtinger 1973, 48). Dieser Familienbegriff ist sehr eng gezogen und schließt nur Lebensgemeinschaften ein, die durch ein biologisches Abstammungsverhältnis gekennzeichnet sind. Immer öfter werden Familien aber als Institutionen aufge- fasst, da sie ebenso wie Institutionen „Systeme von Normen und Werthaltungen“ bilden, „die Verhaltenssicherheit für Menschen bieten" (ÖIF 1999, 15). Zudem bietet die Institution Familie nach Auffassung des Österreichischen Instituts für Familienforschung auch „Sicherheit und Abgrenzung von anderen gesellschaftlichen Bereichen, wie etwa der Arbeitswelt und regelt die Beziehungen zwischen Generationen und Geschlechtern" (ebd.). Auch Beham (1989, 9) betont diesen sozialen Aspekt von Familie. Sie beschreibt Familie als eine Primärgruppe, „deren Mitglieder durch Wir-Bewusstsein und Gefühlsintimität verbunden sind“. Die Familie ist maßgeblich für die Persönlichkeitsentwicklung der einzelnen Personen verantwortlich und zeichnet sich zudem auch durch die Solidarität zwischen ihren Mitgliedern aus.

Die Definitionen von Beham und dem ÖIF (1999) beschränken sich nicht nur auf die konventionelle Vorstellung von Familie. Die Vorstellung der klassischen Kernfamilie, die aus einem verheirateten Paar mit ihren leiblichen Kindern besteht, die gemeinsam in einem Haushalt leben, wäre hier also zu eng gegriffen. Auch andere Lebensformen können die genannten Funktionen der Familie erfüllen. So gibt auch das ÖIF $(1999,15)$ an, dass sich die ,institutionalisierte Verfassung von Familie [...] in ihrer Struktur ändern [kann] und [...] natürlich unterschiedliche Ausprägungen erhalten [kann], die manchmal unter dem Titel der Pluralisierung thematisiert werden." Auch die aktuellen Entwicklungen in Österreich, welche noch aufgezeigt werden sollen, verdeutlichen, dass die Beschränkung auf das konventionelle Familienmodell zu kurz greift. Obwohl das klassische Kernfamilienmodell auch quantitativ noch vorherrschend ist, stellt es nur eines mehrerer Familienkonzepte dar, die alle gleichermaßen eine Berechtigung aufweisen.

Auch die amtliche Statistik hat diese Trends berücksichtigt, weswegen die Statistik Austria folgende Definition von Familie auf ihrer Website anführt: „Nach dem Kernfamilien-Konzept der Vereinten Nationen bilden Ehepaare oder Lebensgemeinschaften mit oder ohne Kinder bzw. Elternteile mit Kindern eine Familie. Kinder sind alle mit ihren beiden Eltern oder einem Elternteil im selben Haushalt lebenden leiblichen, Stief- und Adoptivkinder, die ohne eigene Partner im Haushalt leben und selbst noch keine Kinder haben, unabhängig vom Alter des Kindes. In der Summe der Familien sind somit auch Mütter und Väter mit erwachsenen Kindern enthalten. Söhne und Töchter, die bereits aus dem Elternhaus ausgezogen sind, können nicht berücksichtigt werden. Jede Kernfamilie stellt eine eigene Analyseeinheit dar, unabhängig davon, ob die Kernfamilie mit weiteren Kernfamilien oder zusätzlichen Personen einen Haushalt bewohnt" (Statistik Austria 2015, o.S.). 
Diese Definition schließt die konventionelle Kernfamilie ein, in denen verheiratete Paare mit ihren leiblichen Kindern zusammenleben. Stieffamilien und Familien mit adoptierten Kindern fallen ebenso unter diese Begriffsbestimmung, falls diese im selben Haushalt leben. Ein-Eltern-Familien, d.h. Elternteile, die ihre Kinder alleine erziehen, sind ebenso miteinbezogen. Eine weitere Familienkonstellation wäre die Patchworkfamilie, bei der Elternteile Kinder aus früheren Ehen oder Beziehungen mitbringen. Eine bestehende Ehe oder gemeinsame Kinder sind per definitionem keine Bedingungen mehr, um als Familie zu gelten. Auch verheiratete oder unverheiratete Paare ohne Kinder gelten als Familie, wenn sie gemeinsam in einem Haushalt wohnen. Zudem werden gleichgeschlechtige Paare einbezogen, falls diese in einem gemeinsamen Haushalt leben.

Der Begriff Familie ist also viel umfassender als das Bild des konventionellen Kernfamilienmodells, das oft in unseren Köpfen verankert ist. Doch warum sind andere Familienkonstellationen in unserer Vorstellung nicht so präsent wie das konventionelle Kernfamilienmodell, obwohl sie de facto auch vorhanden sind?

\subsection{Familienkonstellationen im Wandel}

Geprägt wird unser traditionelles Familienbild vor allem durch Sozialisation, Erziehung und Medien, in denen häufig Familien bestehend aus Eltern und Kindern, zumeist zwei, gezeigt werden. Besonders im Kultur- und Medienbereich werden jedoch zunehmend auch alternative Familienkonstellationen, beispielsweise alleinerziehende Mütter oder homosexuelle Paare, gezeigt. So adoptieren zum Beispiel die beiden männlichen Hauptfiguren der Serie „Two and a half Men" einen jungen Burschen. Auch der Musiker Elton John bekennt sich, wie viele andere Personen des öffentlichen Lebens, zu seiner Homosexualität. Trotz dieser steigenden Häufigkeit medialer Darstellung liegt die Präferenz eines konventionellen Kernfamili-

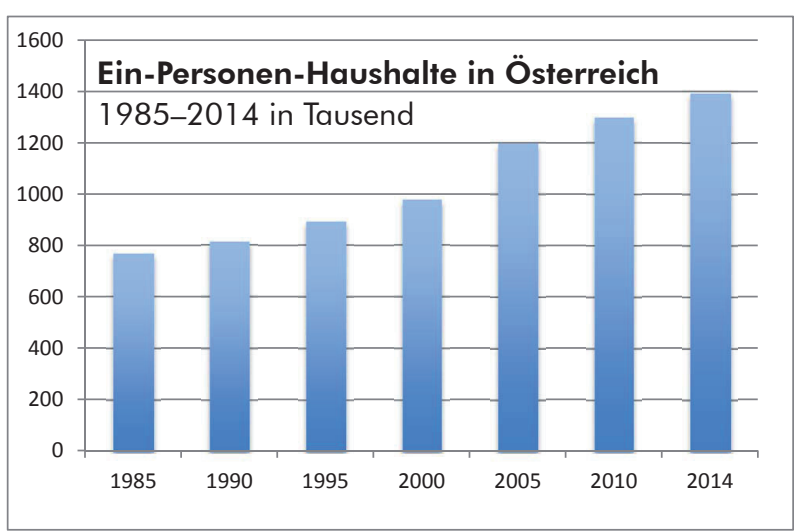

enmodells oft in der eigenen Vergangenheit. Die eigenen Eltern und Großeltern werden zumeist noch als Ehepaar zusammengelebt und mehrere Kinder gehabt haben. Diese Familienkonstellation verankert sich im Unterbewusstsein und stellt somit den Idealtypus einer Familie dar (vgl. Neuwirth 2007).

Laut einer Umfrage des Linzer Market-Institutes gilt das konventionelle Familienbild jedoch in immer weniger österreichischen Haushalten, während andere Familienkonstellationen an Bedeutung gewinnen (vgl. Institut für Markt-, Meinungs-, und Mediaforschung 2015). Konkret äußert sich dies unter anderem darin, dass die Anzahl der Familien ohne Kinder im Zeitraum von 1985 bis 2014 um beinahe ein Drittel stieg. Ebenso wies auch die Anzahl der Ein-Personen-Haushalte einen starken Anstieg auf (vgl. Abb. 1). Ein weiteres Beispiel ist der Verzicht auf Heirat: im Vergleich zu 1985 gab es 2014 fünfmal so viele unverheiratete Paare. Auch die Anzahl der Alleinerziehenden ist seit 1985 um $11 \%$ angestiegen (vgl. Statistik Austria 2015).

In den letzten Jahren wurden auch neue Familienkonstellationen rechtlich anerkannt. Am 1.1.2010 wurde ein neues Rechtsinstitut für gleichgeschlechtliche Paare geschaffen und das Gesetz über die eingetragene Partnerschaft ist in Kraft getreten (vgl. HessKnapp 2010). Unter eingetragener Partnerschaft versteht man eine Lebensgemeinschaft auf Dauer mit gegenseitigen Rechten und Pflichten zwischen zwei Menschen des gleichen Geschlechts. Voraussetzung dafür ist die Volljährigkeit beider Personen, keine aufrechte Ehe, keine aufrechte eingetragene Partnerschaft, keine Verwandtschaft in gerader Linie, keine voll- oder halbbürtigen Geschwister sowie auch kein Adoptivverhältnis (vgl. Bundeskanzleramt Österreich 2015).

Von der österreichischen Politik wurde der Trend Richtung Pluralisierung der Familienkonstellation schon wahrgenommen. Dennoch wird das klassische Familienbild in vielerlei Hinsicht noch als idealtypisch forciert. Daraus können sich Benachteiligungen für andere Familienformen ergeben. Bei Patchworkfami-

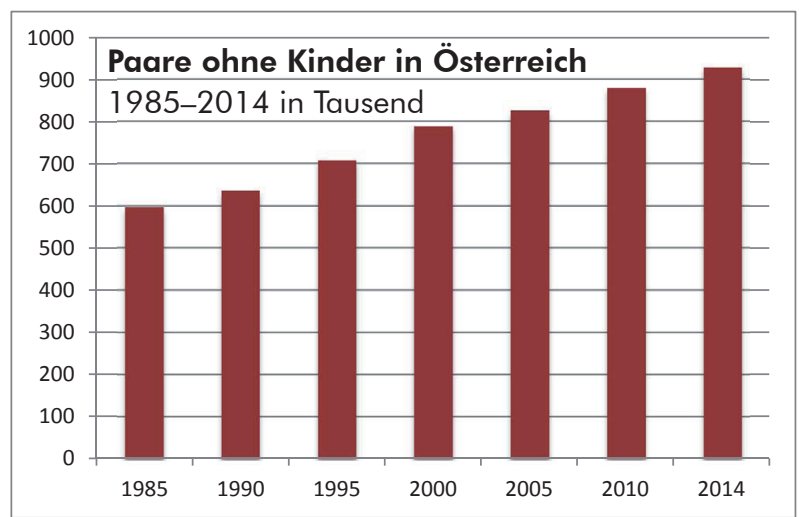

Abb. 1: Entwicklung der Anzahl von Ein-Personen-Haushalten und Paaren ohne Kindern in Österreich von 1985-2014. Datengrundlage: Statistik Austria (2016): Privathaushalte nach Haushaltstypen 1985-2016. https://www.statistik.at/web_de/statistiken/menschen_und_gesellschaft/bevoelkerung/haushalte_familien_lebensformen/haushalte/023300.html(11.10.2016). Gestaltung: Katharina Hörmann 
lien können etwa Probleme in Bezug auf das Erbrecht auftreten. Rechtlich gesehen sind nämlich Stiefkinder nicht als Erben vorgesehen. Um die Kinder als Erben einzusetzen, müsste dies in einem Testament festgehalten werden (vgl. BMWFJ 2011). Auch im Adoptionsrecht wurden bestimmte Familienkonstellationen jahrelang diskriminiert. Homosexuellen Paaren blieb es nach dem Allgemeinen Bürgerlichen Gesetz lange verwehrt zu adoptieren. Dieses Verbot stellte sich aber als verfassungswidrig heraus. Der österreichische Verfassungsgerichtshof fällte im Januar 2015 die Entscheidung, dass es nicht rechtmäßig sei, eingetragene Partner und Partnerinnen nur aufgrund ihrer sexuellen Orientierung von der Adoption eines Wahlkindes auszuschließen. Den Richtern und Richterinnen zufolge widerspreche das Verbot dem Gleichheitsgrundsatz und dem Artikel 8 der Menschenrechtskonvention, welcher besagt, dass jeder Mensch das Recht auf Achtung seines Familienlebens hat. $\mathrm{Zu}$ einer Aufhebung des Verbots kam es zu Beginn des Jahres 2016. (vgl. Matzenberger 2015)

\section{Unterrichtssequenz zum Thema Familien- bilder}

\subsection{Fachdidaktischer $\&$ fachwissenschaftlicher Hintergrund der Unterrichtssequenz}

Im Zuge des fachdidaktischen Praktikums in GW durften wir vier Stunden in einer 1. Klasse HAK im Team abhalten. Diese vier Stunden beschäftigten sich mit dem Thema „Demographischer Wandel in Österreich". In den letzten zwei Stunden dieser Sequenz fokussierten wir uns auf das Thema Familienbilder und deren Implikationen für die moderne österreichische Gesellschaft. Der fachdidaktische und fachwissenschaftliche Hintergrund unserer Planung soll nun vorgestellt werden.

Die Institution Familie besitzt für eine Analyse im GW-Unterricht mehrfach Relevanz. Demographische Sachverhalte und Entwicklungen, die in mehreren Jahrgängen ein Schwerpunktthema bilden (z. B. 5. und 7. Klasse AHS, 1. und 2. Jahrgang HAK; vgl. BMB 2011 bzw. BMB 2014) können auch anhand von Familienverbänden dargestellt werden, da der demographische Wandel nicht selten mit Veränderungen im Familiengefüge einhergeht. Neben demographischen Entwicklungen sind die Familiengründung und ihr erfolgreicher Erhalt auch von ökonomischen Faktoren abhängig. Eine Familie verursacht Kosten und unterliegt besonderen rechtlichen und steuerlichen Regelungen, welche sich als vorteilhaft erweisen können. Die Realisierung unterschiedlicher Familienformen hängt deshalb auch von ihrer Finanzierbarkeit ab.
Die von uns konzipierte Unterrichtseinheit ist daher im Kontext demographischer und sozialökonomischer Lehrinhalte zu sehen. Wenngleich die Sequenz sicher unabhängig abgehalten werden kann, erscheint es doch am sinnvollsten sie etwa im Rahmen bevölkerungsgeographischer Themenstellungen durchzuführen, um entsprechend den Zielen der zu erreichenden Synthesekompetenz (vgl. BMB 2011) zu verdeutlichen, inwiefern eine Institution wie die Familie von gesamtgesellschaftlichen Implikationen und persönlichen Lebenszielen beeinflusst und modifiziert wird.

Bei unserer Themenstellung lassen sich außerdem Synergien zu anderen Unterrichtsfächern, allen voran Geschichte und Sozialkunde, schließen. So könnte man die Formen von Familien anhand unterschiedlicher historischer und geographischer Kontexte betrachten und diese dann vergleichen, um zu sehen, welche Faktoren zu jener Zeit oder an jenem Ort konstituierende Einflüsse auf die einzelnen Familientypen hatten. Bei einer fächerübergreifenden Kooperation mit dem Fach Deutsch würde es sich zum Beispiel empfehlen, einen Familienroman zu lesen und zu interpretieren. Von Herbert Pichler haben wir den Hinweis erhalten, dass sich hierfür die Werke Middlesex von Jeffrey Eugenides (2003) oder Die Korrekturen von Jonathan Franzen (2003) gut eignen würden.

Das Thema Familie lässt sich auf unterschiedlichste Weise bearbeiten und in den Unterricht integrieren. Im vorliegenden Fall haben wir uns für einen konstruktivistischen Unterrichtsansatz entschieden, um den Schülerinnen und Schülern die Möglichkeit zu geben, das traditionelle Konstrukt „Familie“ kritisch zu hinterfragen. Gemeinsam mit den Lernenden wollen wir feststellen, inwiefern die kollektive Wahrnehmung der Familienbilder von historischen sowie medialen Einflüssen geprägt ist. Die Fragestellung, in welchem Ausmaß die entsprechenden Images konstruiert sind und nicht unbedingt mit der Realität korrelieren, ist dabei zentral.

Die Unterrichtseinheit ist also stark von oben genanntem Grundgedanken bestimmt, denn nur durch kritische Auseinandersetzung mit dem Begriff Familie und anderen selbstverständlichen Begrifflichkeiten, kann man sich erst seiner eigenen Konstruktion bewusst werden und diese, wenn nötig, erweitern. Laut Neeb (Neeb 2009, 35) bringen die Lernenden verschiedene Alltagsvorstellungen und Präkonzepte in den Unterricht mit, welche oft nicht mit schulisch anerkanntem Wissen vereinbar sind. Im Zuge des konstruktivistischen Unterrichts sollen sich die Schüler/ innen ihrer eigenen Konstruktionen zunächst bewusst werden. Anschließend haben sie die Möglichkeit ihre Konstruktionen zu überarbeiten bzw. zu erweitern. Ob dies gelingt, liegt aber bei den Schülerinnen und Schülern selbst. Der konstruktivistische Unterricht 
geht von der Grundidee aus, dass ein Individuum seine Konstruktionen nur selbst, aus eigener Motivation heraus, anpassen und überarbeiten kann. Den Lernenden soll keine vermeintlich „allgemein-gültige“ Konstruktion aufgedrängt werden, sondern sie sollen ihre eigenen Vorstellungen und Werthaltungen weiterentwickeln (vgl. ebd.). Dies ist auch beim Thema Familie wichtig. Im Unterricht sollen sich die Schüler/innen durch gezielte Aufgabenstellung ihrer eigenen Vorstellung von Familie bewusst werden. Zudem soll auch ihre Wahrnehmung geschärft werden, dass verschiedene Familienkonstellationen existieren. Es soll aber nicht vermittelt werden, dass es ein „Richtig" und ein „Falsch“ bei den Familienkonstellationen gibt. Jede Familienkonstellation hat ihre Berechtigung und jede und jeder darf sich auch ihr oder sein Bild einer Idealfamilie bewahren.

\subsection{Umsetzung der Unterrichtssequenz}

Im Folgenden soll die zweistündige Unterrichtssequenz zum Thema Familienbilder vorgestellt werden. Diese gliedert sich thematisch in den großen Komplex der Bevölkerungsgeographie ein und kann in mehreren Schultypen behandelt werden:

Lehrplanbezug:

- HAK: 1. Jahrgang

Bevölkerungsgeografie - Demografische Strukturen und Prozesse und ihre sozioökonomischen Wechselwirkungen (BMB 2014, 24).

- AHS: 7. Klasse

Demographische Entwicklung und gesellschaftspolitische Implikationen - die Entwicklung der österreichischen Bevölkerung (zeitliche und räumliche Dimension) darstellen können sowie mögliche Folgen ableiten und beurteilen (BMB 2011, 4).

- HTL: 1. Jahrgang

Bevölkerungsentwicklung und gesellschaftliche Folgerungen; Bevölkerungsstrukturen und -verteilung; Wanderungsbewegungen und Auswirkungen (BMB 2015, 15).

In dieser Unterrichtssequenz steht das kritischemanzipatorische Vermittlungsinteresse im Vordergrund. Dieses zielt darauf ab, die Lernenden zu selbstständigem Denken über bestimmte Sachverhalte $\mathrm{zu}$ animieren. Das Hinterfragen gesellschaftlicher Wertvorstellungen ist dabei wesentlich (vgl. Vielhaber1999). In diesem Sinne ist das Ziel der vorliegenden Unterrichtssequenz, dass sich die Lernenden mit Widersprüchen und Klischees zum Thema Familie auseinandersetzen sowie mit alternativen Familienkonstellationen befassen. Die Schüler/innen blicken im besten Fall über den Tellerrand und dekonstruie- ren gesellschaftliche Normen (vgl. ebd.).

Passend dazu wurden auch die Lernziele recht ähnlich formuliert. Selbstreflexion und die Erkenntnis, dass es nicht „die Wahrheit“ gibt, stehen beim ersten Lernziel „Ich werde mir bewusst, wie mein kognitives Familienbild aussieht und dass jede Person ein anderes hat" im Vordergrund. Ein kritisches Betrachten der Umwelt, der Medien und der politischen, religiösen sowie sozialen Idealvorstellungen soll bei Lernziel 2 „Ich stelle klassische Familienbilder in Frage" erreicht werden. Das Wissen über die Selbstverantwortung soll unter anderem durch das praktische Arbeiten im Lernziel 3 „Ich kann Werbungen kritisch hinterfragen und selbst neue Formen von Werbung entwickeln" vermittelt werden. Die Lernziele sind jeweils für die einzelnen Arbeitsschritte in der Planungsmatrix im Anhang ausgewiesen.

\section{Phase 1:}

Als Einstieg in das Thema des Familienbildes wurde ein Arbeitsauftrag in Einzelarbeit gewählt. Ziel war es, dass sich jede Schülerin oder jeder Schüler bewusst wird, wie ihr oder sein eigenes kognitives Familienbild aussieht und dass jede Person ein anderes besitzt. Die Aufgabenstellung lautete,Zeichne eine Familie“. Auf genauere Angaben wurde bewusst verzichtet, weil jegliche weitere Aufgabenstellung zu einer Beeinflussung geführt hätte. Auch auf Fragen wie „Darf es auch eine homosexuelle Familie sein?“ und „Darf auch der Hund mit drauf?", antworteten wir stets mit „Eine Familie - wie auch immer die aussieht". Die Panik, die viele Schülerinnen und Schüler beim Wort „Zeichnen“ überkam und die Rufe „Ich kann aber nicht schön zeichnen!"verschwanden bzw. verstummten, als wir darauf hinwiesen, dass keinesfalls die Ästhetik des Bildes im Vordergrund steht. Nach der Einzelarbeit hatten die Schülerinnen und Schüler die Möglichkeit, ihre Bilder kurz zu präsentieren und miteinander zu vergleichen sowie Unterschiede zu diskutieren. Auch wir Studentinnen und Studenten hatten zuvor Bilder gezeichnet, die bewusst nicht-klassische Familienkonstellation zeigten, beispielsweise ein Pärchen ohne Kinder, ein alleinerziehender Vater mit Kind oder ein homosexuelles Paar mit Kindern, um den Schülerinnen und Schülern zu zeigen, dass neben klassischen Familienkonstellationen auch weitere existieren. Die Vielfalt gegenwärtiger Familienkonstellationen wurde in der Plenumsdiskussion besonders hervorgehoben. Anschließend bekamen die Schülerinnen und Schüler das Arbeitsblatt 1 (siehe Anhang) mit Fragen zu ihren gezeichneten Bildern, um die Ergebnisse zu festigen. Auch das Interpretieren von Statistiken sollte geübt werden. Durch die Frage 3 zur eigenen zukünftigen Familie sollen die Schüler/innen erkennen, dass möglicherweise die Vorstellung einer Familie gar nicht 
zum persönlichen Plan der zukünftigen Familie passt. Auch diese Ergebnisse wurden im Plenum verglichen.

\section{Ergebnisse der Schüler/innen:}

Von den Lernenden wurden zum größten Teil (rund die Hälfte) Familien mit Vater und Mutter sowie zwei bis drei Kinder gezeichnet. Etwa ein Viertel der Schüler/innen zeichnete Vater und Mutter mit entweder einem Kind oder vier Kindern. Das restliche Viertel der Klasse zeichnete Familienbilder, in denen beispielsweise auch der Hund, die Großeltern sowie Tanten und Onkeln zu sehen waren. Nur ein Schüler zeichnete eine Familie mit einer alleinerziehenden Mutter und eine Schülerin entschied sich für ein homosexuelles Pärchen mit Kind (vgl. Abb. 2).

Auf manchen Bildern zeichneten die Schüler/innen die Familien, in denen sie selbst momentan lebten, jedoch war dies nicht immer der Fall. Interessant war, dass besonders jene, die Familien mit drei oder mehr Kindern gezeichnet hatten, antworteten, dass sie selbst nicht so eine große Familie haben möchten, sondern nur ein bis zwei Kinder haben wollen.

\section{Phase 2:}

In der zweiten Phase der Unterrichtssequenz wird im Zuge einer Gruppenarbeit die mediale Darstellung des Begriffs Familie analysiert und diskutiert. Die kritische Rezeption von Werbungen, mit der die Lernen- den beauftragt werden, steht hierbei im Vordergrund und soll dazu führen, dass die Jugendlichen öffentlich präsentierte Familienbilder sensibler und bewusster wahrnehmen. Dazu erledigen die Lernenden in Gruppenarbeiten mehrere Aufgaben, welche sich auf drei Bildimpulse beziehen (vgl. Arbeitsblatt 2). Alle gewählten Bilder am zweiten Arbeitsblatt zeigen heterosexuelle Elternpaare mit mehreren Kindern. Diese Darstellungen wurden gewählt, da sie in der Werbung allgegenwärtig sind und so die gesellschaftliche Norm suggerieren. Von besonderer Relevanz war es daher, dass die Lernenden im Zuge eines Arbeitsauftrages diskutieren, inwieweit die medialen Familiendarstellungen der realen Vielfalt gerecht werden. Die Zuordnung zu einem ungefähren zeitlichen Rahmen soll die Basis für eine Diskussion über die Änderung des Familienbildes bzw. einer Rollenverteilung bieten, da sie zeigt, dass noch immer sehr ähnliche mediale Familienbilder wie vor fünfzig Jahren vorherrschen. Schließlich wurden die Schüler/innen dazu animiert, neue Formen von Werbungen zu kreieren, welche unterschiedlichste Familienkonstellationen miteinschließen. Dazu konnten sie eine der abgebildeten oder andere Werbungen mittels Photoshop überarbeiten oder schauspielerisch darstellen. Auch andere Darstellungsmöglichkeiten konnten gewählt werden. Genauere Vorgaben darüber, wie diese Werbung gestaltet werden sollte, wurden nicht gegeben, um der Kreativität keine Grenzen zu setzen.

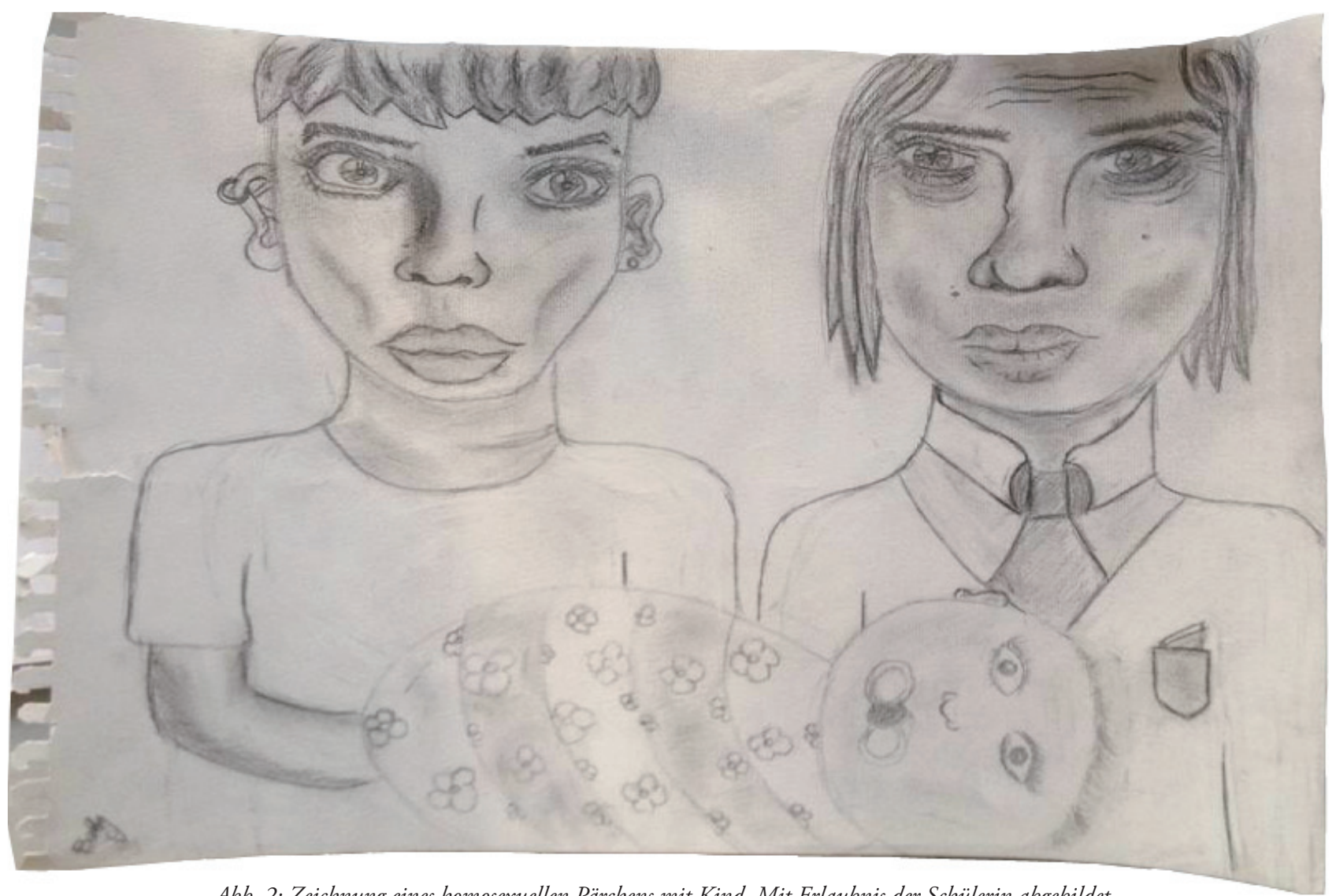

Abb. 2: Zeichnung eines homosexuellen Pärchens mit Kind. Mit Erlaubnis der Schülerin abgebildet. 
Ergebnisse der Schüler/innen:

Die Zeiteinteilung und die Diskussion zu den Familienbildern und damit verbundene Klischees fielen den meisten Schülerinnen und Schülern relativ leicht. Bei den umgestalteten oder neu gestalteten Werbungen entschieden sich einige Schüler/innen dazu, das Thema Homosexualität einzubauen. Eine Gruppe bearbeitete ein Bild einer Familie aus Vater, Mutter und Kind so, dass das Kind zwei Väter hatte und warb damit für eine Adoptionsagentur. Auch Ein-ElternFamilien wurden in den Werbungen aufgegriffen. Beispielsweise spielte eine Gruppe eine Werbung für die Anti-Baby-Pille, in der eine alleinerziehende Mutter meint, dass zwei Kinder genug sind. Eine weitere Gruppe performte einen erfundenen Werbespot, in der eine alleinerziehende Mutter ganz angetan war von ihren neuen Ohrstöpseln, weil sie mit ihnen die Streitereien ihrer Kinder nicht mehr hören konnte.

Vor den Präsentationen wurde vereinbart, dass sich die Gruppen nach einem Rotationsprinzip gegenseitig Feedback geben. So wurde zum einen der Arbeitsauftrag erfüllt und zum anderen auch das Geben von konstruktiver Kritik geübt. Auch die Lehrenden konnten den Lernenden an dieser Stelle mündliches Feedback überbringen.

Unterrichtsende:

Die Stunde wurde mit einer gemeinsamen Reflexion im Plenum abgeschlossen. Die zentrale Frage war hierbei, inwiefern die Dekonstruktion der Familienbilder in den GW-Unterricht und zum Thema „Bevölkerung in Österreich“ passt. Anders gefragt: „Warum bearbeiten wir das Thema überhaupt?" Die Lernenden antworteten, dass nicht nur das konventionelle Familienbild in der Bevölkerung in Österreich gelebt wird, sondern auch alternative Formen des $\mathrm{Zu}$ sammenlebens existieren. Diese sollten im Sinne der Gleichberechtigung ein Teil des Unterrichts sein.

Als sehr kritischer Punkt wurde von einem Schüler angemerkt, dass dieses Thema in keinem anderen Unterrichtsfach Platz findet und somit nur der GW-Unterricht übrig bleibt. Wir Studentinnen und Studenten finden jedoch, dass in vielen anderen Fächern ebenso die Möglichkeit bestünde, dieses Thema zu behandeln. Von unserer Seite aus wurde zusätzlich angemerkt, dass wir sie für dieses Thema auch sensibilisieren wollten, um ihnen zu zeigen, dass es bei der Frage nach Familienkonstellationen weder ein Richtig noch ein Falsch gibt.

\section{$4 \quad$ Reflexion und Fazit}

Die Ergebnisse der durchgeführten Unterrichtssequenz verdeutlichen, dass die Lernenden mit dem Begriff Familie häufig jene Modelle assoziieren, die auch ein Großteil der österreichischen Gesellschaft damit in Verbindung bringt, nämlich jene von Ehepaaren mit (zumeist zwei) leiblichen Kindern. Wie im zweitenKapitel dieses Artikels dargestellt wird, ist dieses Familienmodell quantitativ immer noch vorherrschend. Dennoch ist das konventionelle Kernfamilienmodell nur eine mögliche Realisationsform von Familie.

Die Umsetzung der Unterrichtssequenz hat insgesamt sehr gut funktioniert. Die Motivation der Jugendlichen war durchgehend hoch, was wir unter anderem darauf zurückführen, dass das Thema sich sehr an der Lebenswelt der Schüler/innen orientiert. Wir meinen, dass unsere wesentlichsten Anliegen durch die vorliegende Unterrichtsreihe vermittelt werden, nämlich, dass das klassische Familienbild in unserer modernen Konsumgesellschaft einem stetigen Wandel ausgesetzt ist und dass Medien bestimmte Werthaltungen und Leitbilder präferieren, die man kritisch reflektieren sollte. Die Schüler/innen hatten im Zuge der Unterrichtssequenz die Möglichkeit, sich ihrer eigenen Vorstellung von Familie bewusst zu werden und darüber zu reflektieren. Zudem konnten sie auch ihre Wahrnehmung bezüglich anderer Familienkonstellationen erweitern. Besonders das Auswerten der Statistiken zu den Familienkonstellationen war hierbei ein wichtiger Schritt, da die Lernenden feststellen konnten, wie repräsentativ ihre kognitiven Familienbilder tatsächlich sind. Die Schüler/innen gaben mehrmalig das Feedback, dass sie nicht erwartet hätten, dass die Ein-Kind-Familie in Österreich die am häufigsten auftretende Form ist. Viele Lernende rechneten damit, dass Familien mit zwei bis drei Kindern öfter vorkommen würden. Des Weiteren berichteten viele Schülerinnen und Schüler, dass sie nicht wussten, dass auch Paare ohne Kinder von der Statistik Austria als Familie gezählt werden.

Die Umsetzung der zweiten Unterrichtsphase, in welcher sich die Schüler/innen mit medialen Repräsentationen der Familie auseinandersetzten, funktionierte ebenfalls gut. Unsere Intention war hierbei, den Schülerinnen und Schülern bewusst zu machen, dass sie durch ihr Handeln und Denken die Schaffenden der zukünftigen Familienkonstellationen sind, beispielsweise als Elternteile, Lehrende, Mediengestaltende oder ähnliches. In ihren gezeichneten Familienbildern oder den selbst erstellten Werbungen stellten die Lernenden auch alternative, neue Familienkonstellationen (z. B. ein homosexuelles Pärchen mit Kind, alleinerziehende Mütter, etc.) dar, die erst in der jüngeren Vergangenheit an Bedeutung gewonnen haben. Das zeigt, dass die Schüler/innen derartige Veränderungen bewusst wahrnehmen und ihnen zumeist mit sehr offener Haltung gegenüberstehen. Hieran könnten sich manche reaktionäre Menschen durchaus ein Beispiel nehmen. 
Da die Klasse leider nur zwei Wochen von uns Studentinnen und Studenten begleitet wurde, war es nicht möglich zu beurteilen, ob ein bleibendes Bewusstsein dafür geschaffen wurde, dass sozial konstruierte Familienbilder einer kritischen Auseinandersetzung bedürfen. Die Ergebnisse der Lernenden weisen jedoch darauf hin, dass ihnen durch die (de-) konstruktivistische Aufgabenstellung zumindest im Zuge der Unterrichtssequenz eine entsprechende Sensibilisierung widerfahren ist.

\section{Literatur}

Beham, M. (1989): Diskussion des Begriffs Familie. In: Gisser, R. (Hrsg.):LebensweltFamilie: Familienbericht 1989,9-12. Wien: Bundesministerium für Gesundheit und Frauen.

Bundeskanzleramt Österreich (2015): Allgemeines zur eingetragenen Partnerschaft. https://www.help.gv.at/Portal. Node/hlpd/public/content/189/Seite.1890100.html (24.10.2016).

Bundesministerium für Bildung (Hrsg.) (2011): Lehrplan AHS-Oberstufe. https://www.bmbf.gv.at/schulen/ unterricht/lp/lp_neu_ahs_06_11858.pdf (24.10.2016).

Bundesministerium für Bildung (Hrsg.) (2014): Lehrplan Handelsakademie.http://www.abc.berufsbildendeschulen. at/upload/598_HAK\%20LP\%202004\%20-\%20 Anlage\%201.pdf (24.10.2016).

Bundesministerium für Bildung (Hrsg.) (2015): Lehrplan Höhere Technische Lehranstalten. http://www.eduhi.at/ dl/HTL_BGBl_II_Nr_300_2011_07_Sept_Anlage_1. pdf (24.10.2016).

Bundesministerium für Wirtschaft, Familie und Jugend (Hrsg.) (2015): Patchwork-Familie. Ein Ratgeber und Begleiter für Familien. http://www.bmf.gv.at/dam/ jcr:d66685ed-03d8-43c5-8491-13a91d4d7a75/patchwork.pdf (24.10.2016).

Euginides, G. (2003): Middlesex. Reinbek bei Hamburg: Rowohlt Verlag.
Franzen,J. (2003): Die Korrekturen. Reinbek bei Hamburg: Rowohlt Verlag.

Feichtinger, G. (1973): Bevölkerungsstatistik. Berlin: De Gruyter.

Hess-Knapp, H. (2010): Gleiches Recht für alle? In: Arbeiterkammer (AK) und Österreichischer Gewerkschaftsbund (ÖGB) (Hrsg.): Arbeit \& Wirtschaft. http://www.arbeit-wirtschaft. at $/$ servlet $/$ ContentServer? pagename $=$ X03/Page $/$ Ind ex\&n=X03_1.a_2010_07.a\&cid $=1280336442012$ (24.10.2016).

Lüscher, K. (O.J.): Familie im gesellschaftlichen Wandel, unveröffentlichtes Manuskript.

Matzenberger, M. (2015): Schwule und Lesben sollen auch fremde Kinder adoptieren dürfen. In: Der Standard, 14.01.2015. http://derstandard.at/2000010361671/ Schwule-und-Lesben-bekommen-Adoptionsrecht (24.10.2016).

Neeb, K. (2009): Chancen und Grenzen eines konstruktivistischen Unterrichtsversuchs im schulischen Alltag. In: GW-Unterricht 116, 29-46.

Neuwirth, N. (Hrsg.) (2007): Familienformen in Österreich: Stand und Entwicklung von Patchwork- und EinEltern-Familien in der Struktur der Familienformen in Österreich. Wien: Österreichisches Institut für Familienforschung.

Österreichisches Institut für Familienforschung (ÖIF) (Hrsg.) (1999): Österreichischer Familienbericht. (= Österreichischer Familienbericht 4). http://www. oif.ac.at/fileadmin/OEIF/andere_Publikationen/fb99_ band1.pdf (24.10.2016).

Statistik Austria (Hrsg.) (2015): Familien. http://www.statistik.at/web_de/statistiken/menschen_und_gesellschaft/ bevoelkerung/haushalte_familien_lebensformen/familien/index.html (30.12.2015).

Vielhaber, C. (1999): Türen öffnen im GW-Unterricht. In Vielhaber, C. (Hrsg.): Fachdidaktik Kreuz und Quer (=Band 15 der Materialien zur Didaktik der Geographie u. Wirtschaftskunde). Wien: Institut für Geographie und Regionalforschung, 9-26. 
Ablauf der Unterrichtseinheiten

\begin{tabular}{|c|c|c|c|}
\hline Zeit & Ablauf & Sozialform / Methode & Lernziele \\
\hline \multicolumn{4}{|c|}{ Phase 1} \\
\hline \multirow[t]{2}{*}{15 Min. } & $\begin{array}{l}\text { Arbeitsauftrag: "Zeichne eine } \\
\text { Familie!" }\end{array}$ & Einzelarbeit & \multirow{2}{*}{$\begin{array}{l}\text { Ich werde mir bewusst, wie mein kognitives } \\
\text { Familienbild aussieht und dass jede Person ein } \\
\text { anderes hat. } \\
\text { Ich stelle klassische Familienbilder in Frage. }\end{array}$} \\
\hline & $\begin{array}{l}\text { Präsentation und Diskussion } \\
\text { der Bilder im Plenum }\end{array}$ & Plenumsdiskussion & \\
\hline \multirow[t]{3}{*}{15 Min. } & $\begin{array}{l}\text { Beantwortung der Fragen auf } \\
\text { Arbeitsblatt } 1\end{array}$ & Einzelarbeit & \multirow{2}{*}{$\begin{array}{l}\text { Ich werde mir bewusst, wie mein kognitives } \\
\text { Familienbild aussieht und dass jede Person ein } \\
\text { anderes hat. } \\
\text { Ich stelle klassische Familienbilder in Frage. }\end{array}$} \\
\hline & $\begin{array}{l}\text { Vergleichen der Ergebnisse im } \\
\text { Plenum }\end{array}$ & Plenumsdiskussion & \\
\hline & \multicolumn{3}{|c|}{ Phase 2} \\
\hline 30 Min. & $\begin{array}{l}\text { Arbeitsblatt 2: Schüler/innen } \\
\text { erledigen zunächst Aufgaben } \\
\text { 1-4 am Arbeitsblatt und kreie- } \\
\text { ren eine eigene Werbung, wel- } \\
\text { che sie anschließend präsentie- } \\
\text { ren (Aufg. 5) }\end{array}$ & Gruppenarbeit & $\begin{array}{l}\text { Ich stelle klassische Familienbilder in Frage. } \\
\text { Ich kann Werbungen kritisch hinterfragen und } \\
\text { selbst neue Formen von Werbung entwickeln. }\end{array}$ \\
\hline 30 Min. & $\begin{array}{l}\text { Schüler/innen präsentieren ihre } \\
\text { Werbungen } \\
\text { ( } 5 \text { Min pro Gruppe, } 3 \text { Min Prä- } \\
\text { sentation und } 2 \text { Min Feedback) }\end{array}$ & Präsentation im Plenum & $\begin{array}{l}\text { Ich stelle klassische Familienbilder in Frage. } \\
\text { Ich kann Werbungen kritisch hinterfragen und } \\
\text { selbst neue Formen von Werbung entwickeln. }\end{array}$ \\
\hline 10 Min. & Gemeinsame Reflexion & Plenumsdiskussion & $\begin{array}{l}\text { Ich werde mir dem Ziel der Stunde bewusst. Ich } \\
\text { kann die Entscheidung der Lehrenden, dieses } \\
\text { Thema gewählt zu haben, nachvollziehen. }\end{array}$ \\
\hline
\end{tabular}




\section{Arbeitsblatt Familienbild 1 (Einzelarbeit)}

1. Erläutere, warum du genau dieses Motiv gezeichnet hast.

2. Beschreibe deine Gedanken/die ersten Eindrücke, die du hattest, als die Studierenden ihre gezeichneten Bilder zeigten.

3. Hinterfrage, ob deine momentane Familie mit der gezeichneten übereinstimmt.

4. Gestalte deine Zukunft: Soll deine zukünftige Familie so aussehen wie die von dir gezeichnete Familie?

5. Überprüfe, ob dein gezeichnetes Bild ein repräsentatives Familienbild für Österreich ist. (vgl. Abb. 1) Erläutere dein Ergebnis.
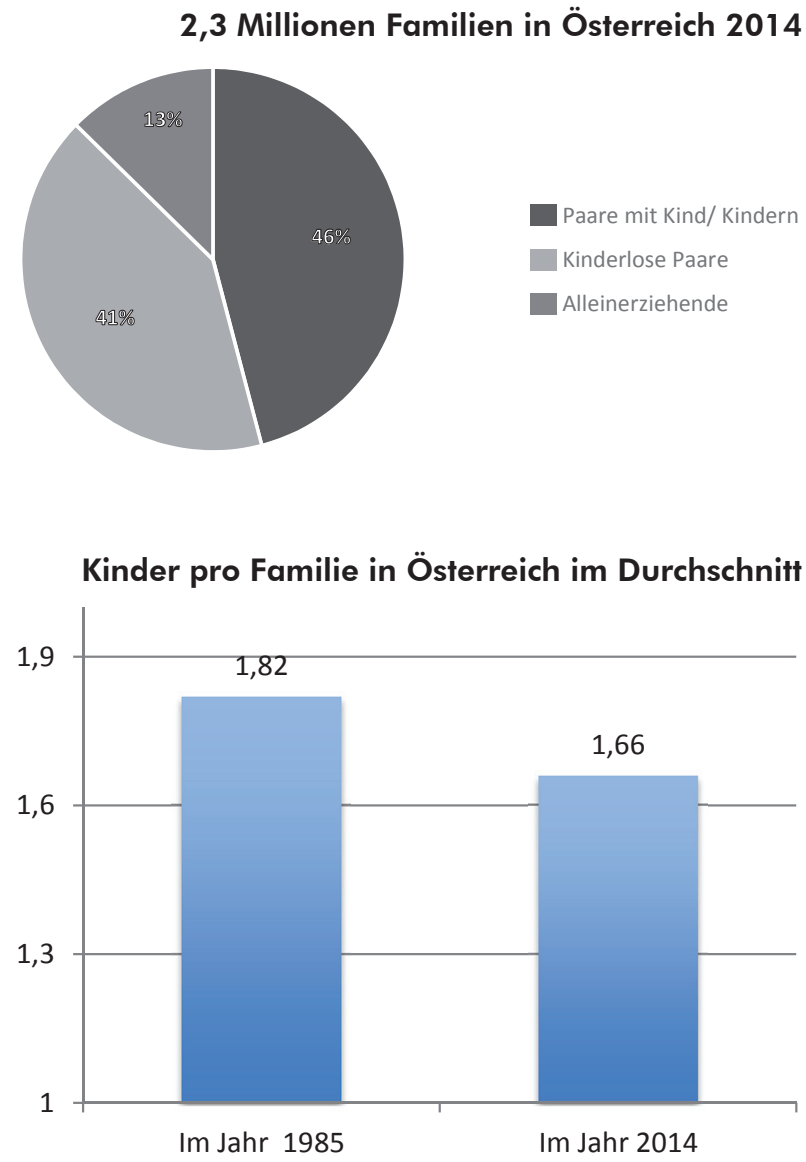

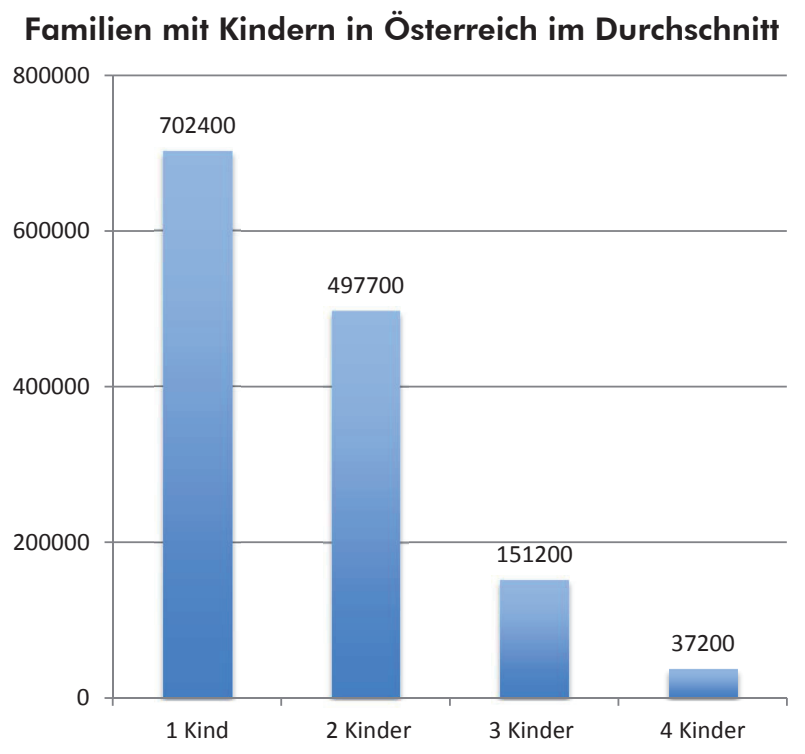

Datengrundlage: Statistik Austria (2015): Familien. https://www.statistik.at/web_de/statistiken/menschen_und_gesellschaft/bevoelkerung/haushalte_familien_lebensformen/familien/ index.html (30.10.2015).

Gestaltung: Katharina Hörmann 


\section{Gruppenarbeit: Woher stammen unsere Familienbilder?}

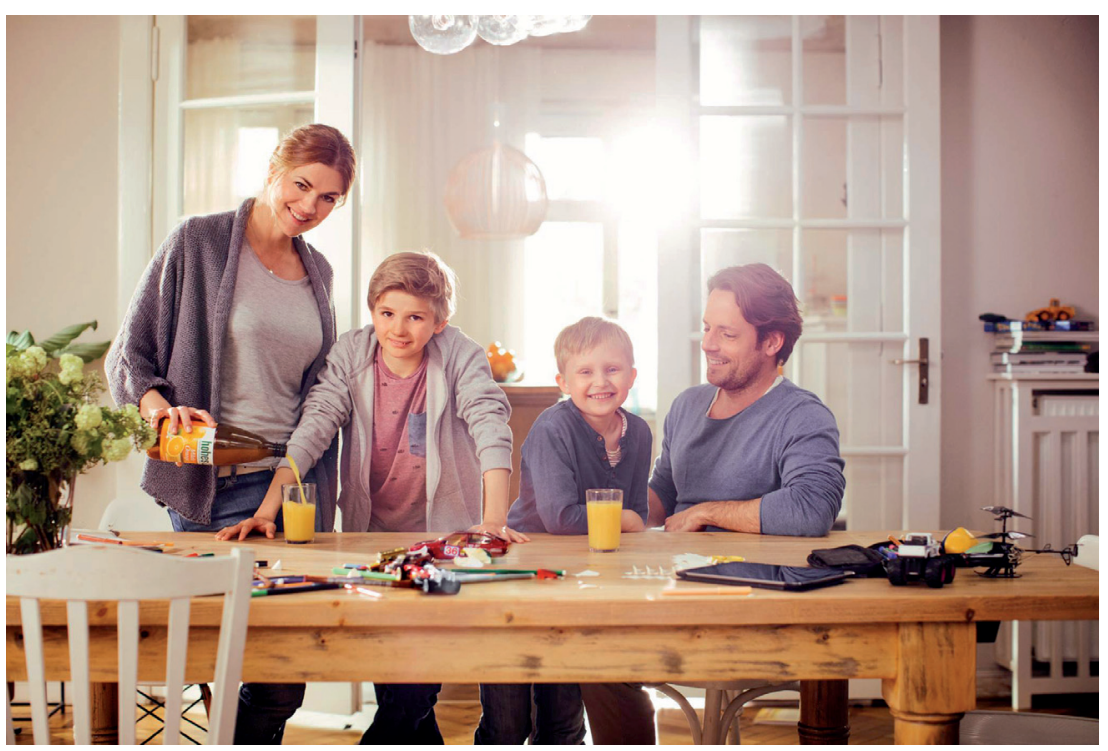

Werbung von hohes $C$

(c) hohes C/ Eckes Granini Deutschland GmbH

\section{Arbeitsblatt Familienbild 1 (Einzelarbeit)}

\section{...und jetzt kommt ein DR.oetKer Pudding!}

1. Ordnet die Fotos einer bestimmten Zeitperiode zu.

2. Erläutert die Gemeinsamkeiten und Unterschiede der vorliegenden Familienbilder.

3. Diskutiert die in den Bildern vorkommenden Stereotype/Klischees.

4. Erklärt, wie sich die Bevölkerungsstruktur entwickeln würde, wenn jede Familie so aussehen würde wie auf den Bildern. Diskutiert, ob diese Bilder der Wirklichkeit entsprechen.

5. Entwerft eine neue Interpretation (z.B. Zeichnen, Photoshop, Beschreibung, Vorspielen etc.) EINER Werbung!
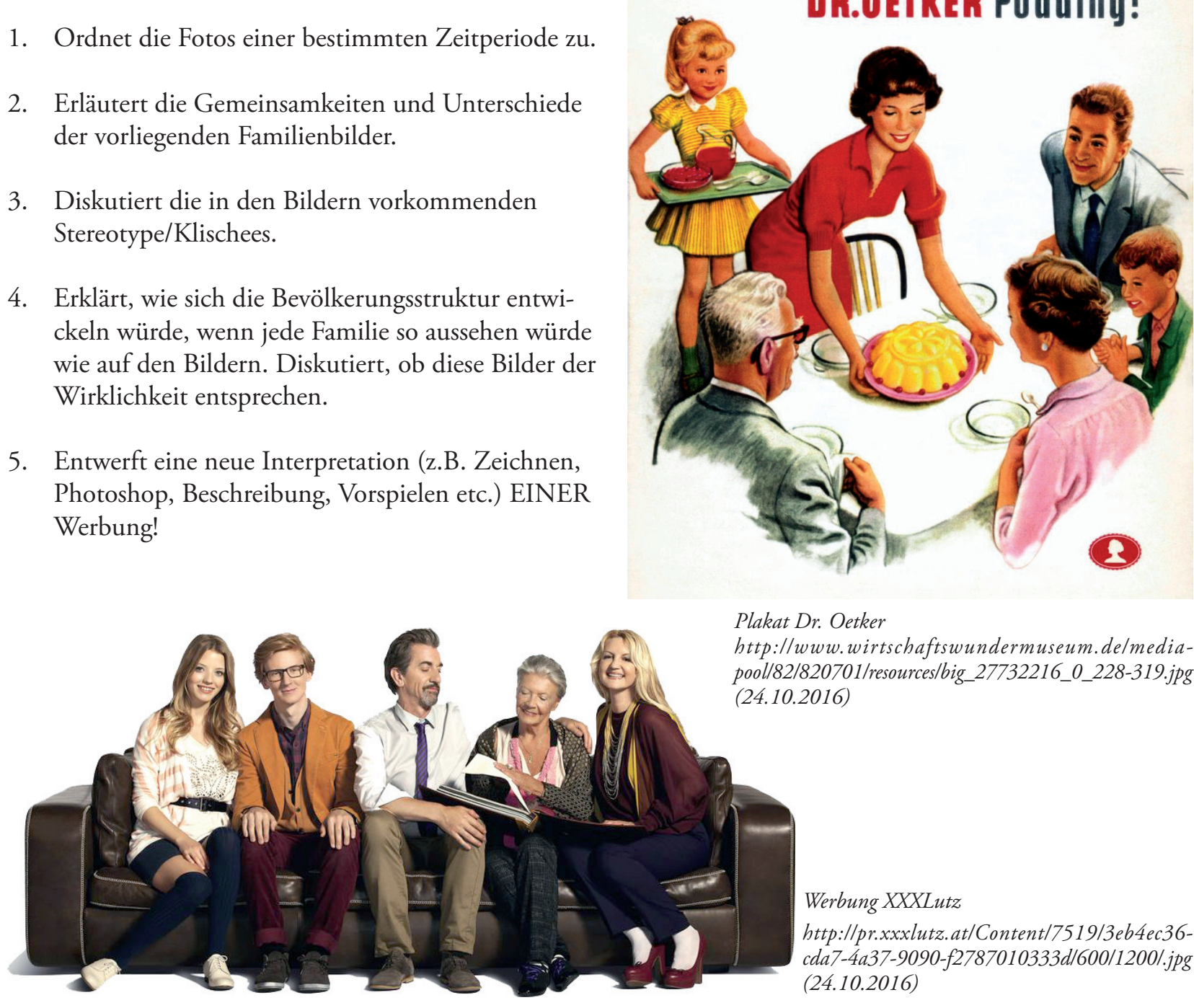

Plakat Dr. Oetker www. wirtschaftswundermuseum. delmediapool/82/820701/resources/big_27732216_0_228-319.jpg (24.10.2016) 